\title{
Développement et utilisation d'applications complémentaires dans une approche SCADA avec une solution communicante Bluetooth
}

\author{
Pascal Vrignat ${ }^{1}$, Florent Duculty ${ }^{1}$, Manuel Avila ${ }^{1}$, Stéphane Begot ${ }^{1}$, Toufik Aggab ${ }^{1}$, Jean- \\ François Millet ${ }^{1}$, David Delouche ${ }^{2}$, Frédéric Kratz ${ }^{3}$ \\ ${ }^{1}$ Université d'Orléans, IUT de l'Indre, Laboratoire PRISME, Châteauroux, France \\ ${ }^{2}$ HEI Campus Centre, Laboratoire PRISME, Châteauroux, France \\ ${ }^{3}$ INSA Centre Val de Loire, Laboratoire PRISME, Bourges, France \\ pascal.vrignat@univ-orleans.fr
}

\begin{abstract}
RESUME : Depuis quelques années, une des thématiques de recherche développée dans le laboratoire PRISME en collaboration avec des partenaires industriels locaux concerne l'estimation du niveau de dégradation d'un processus à l'aide d'un Modèle de Markov Caché $(M M C)$ à partir des informations issues du service de maintenance. Pour cela, l'accès aux données associées à un processus en ligne, à un instant "souhaité", et la sélection des informations les plus pertinentes dans un volume toujours plus important sont nécessaires et utiles pour le développement d'une entreprise. Dans le cadre de la collecte des informations à partir d'un réseau informatique industriel multicouches et de l'utilisation d'outils logiciels appropriés, nous avons mis en place au sein de nos formations un module intitulé "Supervision". Ce module aborde entre autres, les problèmes rencontrés autour d'un serveur $O P C^{l}$ et la connexion au processus à surveiller par différents utilisateurs à partir de technologies diverses. Cet article propose de nombreuses pistes de travail à proposer à des étudiants en formation $B A C+2$ à $B A C+5$ dans le concept de l'usine 4.0.
\end{abstract}

Mots clé : usine 4.0, technologie Bluetooth, SCADA, Serveur OPC, Excel, Matlab

\section{INTRODUCTION}

Dans le contexte économique actuel, les entreprises de biens et de services essayent d'apporter des solutions en matière de prospection autour de leurs outils de production. Certaines entreprises comme par exemple, EDF, Total, Michelin, Orange ou la SNCF tentent de se fédérer afin de mener ensemble, une réflexion prospectiviste. A ce titre, nous pouvons citer EXERA (Association des Exploitants d'Equipements de Mesure, de Régulation et d'Automatisme) qui depuis sa création en 1974 par le ministère de l'industrie, regroupe des industriels de domaines variés (énergie, transport...). Cette association apporte à ses membres, une aide pratique concernant par exemple, des orientations de solutions techniques à adopter en fonction d'un besoin spécifique. D'autres entreprises mènent cette réflexion en interne.

\footnotetext{
${ }^{1}$ OPC : OPCFOUNDATION., https://opcfoundation.org/. : OPC est similaire à DDE (Dynamic Data Exchange) dans l'objectif de faire communiquer de façon transparente différents systèmes ou applications. Dans ses performances, OPC surclasse de loin DDE (contrôle de la qualité des échanges, gestion des erreurs de communication...) qui n'a jamais connu de véritable essor dans le monde industriel. De plus, OPC permet de gérer de façon simple des architectures réseau "Client-Serveur" grâce à des mécanismes natifs dans Windows 95/98/NT : OLE (Object Linking and Embedding), COM (Component Objet Model), DCOM (Distributed Component Objet Model).
} 
Bonduelle, par exemple, a mis en place une réflexion sur ce que sera l'alimentation végétale en 2025. De nombreux indicateurs nous montrent que nous sommes à l'aube d'une révolution majeure, porteuse de nombreuses innovations et créatrice d'une nouvelle dynamique de marché. Plusieurs termes peuvent nommer cette révolution [1] : "Cyber-Usine", "Usine digitale", "Integrated Industry", "Innovative Factory" ou "Industrie 4.0". Le moyen pour y parvenir impliquera obligatoirement les technologies de l'Internet dans un processus de fabrication [2] (Fig. 1). Des objets communicants et autonomes viendront se greffer à la "toile" pour créer un écosystème informationnel utilisant le concept de 1' "Internet des objets" ou "Internet of things".

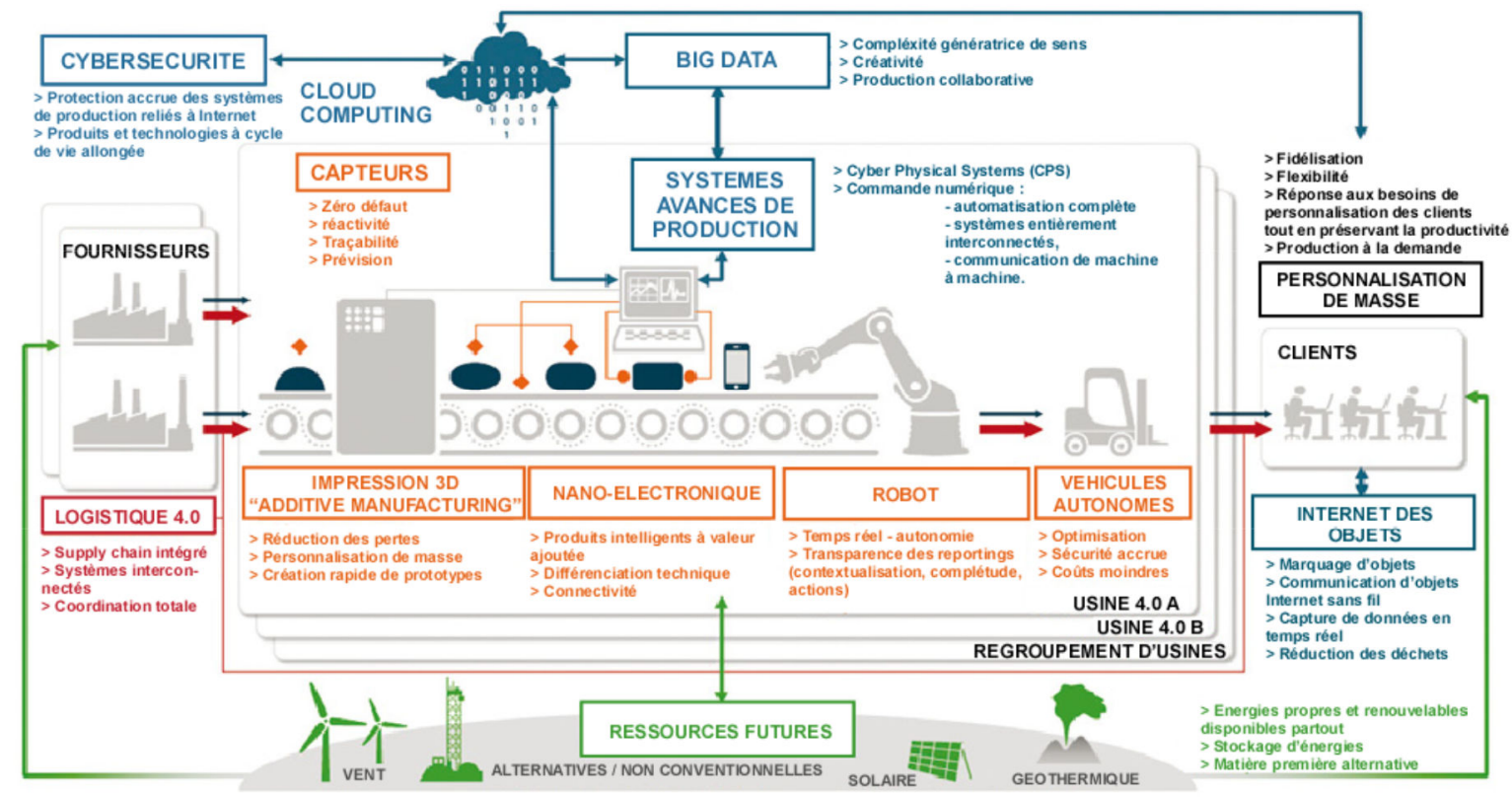

Fig. 1 : Les évolutions qui vont progressivement conduire à l'émergence de l'industrie 4.0

Cette révolution technologique n'est pas sans impact sur nos modèles de pensée et les enseignements que nous devons distiller devant des étudiants de licence de deuxième année à master deuxième année. Cet article se décompose en deux sections. Dans la première section, nous proposons un rapide historique concernant l'évolution de l'industrie pour aboutir à l'industrie 4.0. Dans la seconde section, nous présentons les travaux pouvant être menés dans ce cadre avec des étudiants d'IUT ${ }^{2}$ ou d'écoles d'ingénieurs à vocation technologique. Nous terminons nos propos par une conclusion.

\section{LES REVOLUTIONS INDUSTRIELLES}

La première révolution industrielle repose sur le charbon, la métallurgie, le textile et la machine à vapeur. A la fin du XVIII ${ }^{\text {ème }}$ siècle (début du XIX ${ }^{\text {ème }}$ siècle), arrivent la première machine à tisser mécanique avec moteur hydraulique, le premier essai d'une locomotive à vapeur (1804)... La deuxième révolution industrielle a démarré à la fin du XIX ${ }^{\mathrm{ème}}$ siècle. Elle trouve ses fondements dans l'électricité, la mécanique, le pétrole, les premiers moyens de

\footnotetext{
${ }^{2}$ IUT : Institut Universitaire de Technologie
} 
communication : le télégraphe et le téléphone. Parallèlement à cette époque, Frederick Taylor invente en 1911, le taylorisme, et Henry Ford instaure le montage à la chaîne sur les lignes de production. La troisième révolution industrielle se produit au milieu du $\mathrm{XX}^{\text {ème }}$ siècle, avec une dynamique liée à l'électronique, les télécommunications et l'informatique. Avec l'électronique, c'est l'arrivée du transistor et du microprocesseur. A cette période, deux produits ont impacté la production industrielle : le robot (1959) et l'automate programmable industriel (1968). Ces deux technologies ont été créées par des Américains (Georges Devol et Joseph Engelberger pour le robot, Richard Morley pour l'automate programmable industriel (API)). La quatrième révolution industrielle prend forme sous nos yeux [2], (Fig. 2). Nous pouvons la résumer par la numérisation poussée à l'extrême dans "un monde technologique intelligent : The Smart World". Le terme "Smart" sera employé dans tous les domaines : production énergétique, véhicule, bâtiment... Avec l'industrie 4.0, il y aura une interaction entre les produits fabriqués et les machines. Cette interaction sera d'autant plus opérationnelle et fiable, que l'acquisition des données provenant du terrain en temps réel sera robuste.

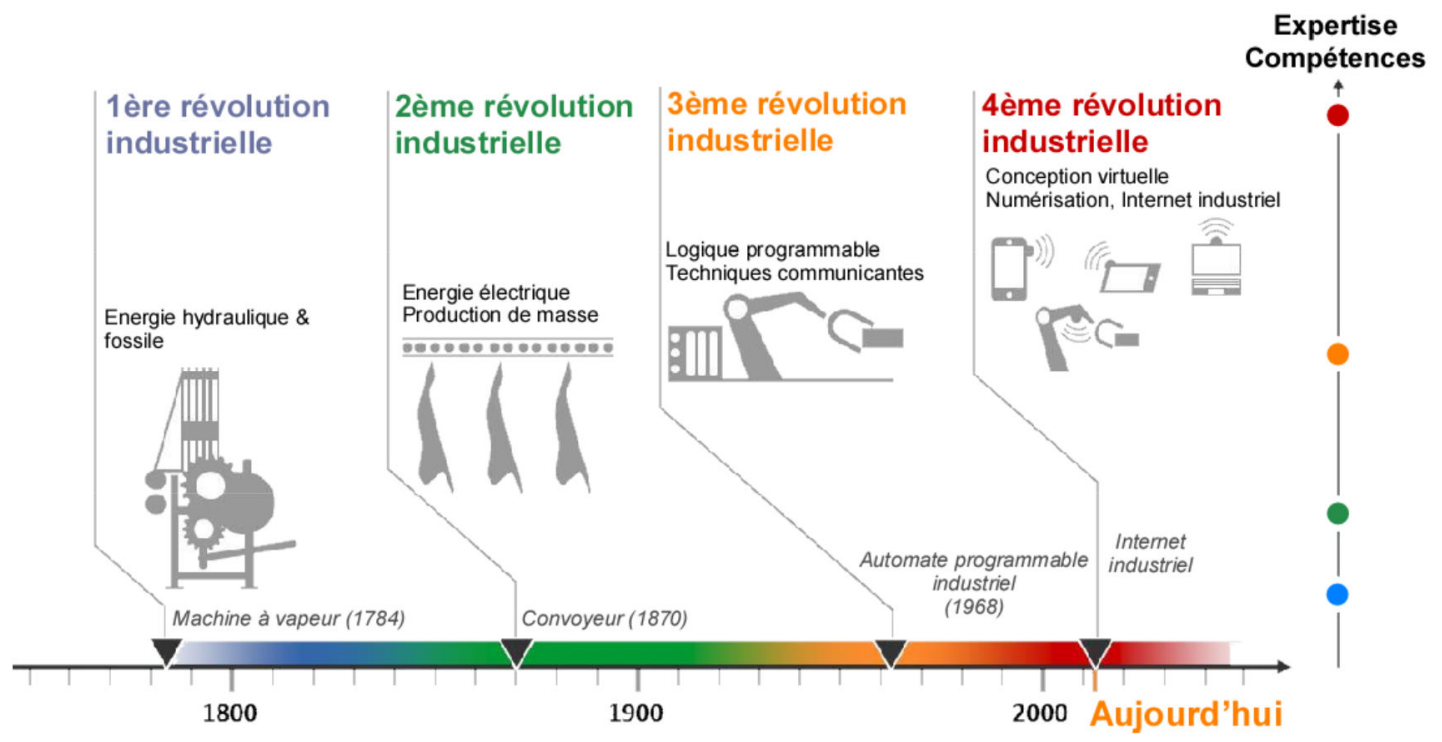

Fig. 2 : Evolution des révolutions industrielles à horizon 2020

Notre environnement deviendra "cliquable" dans un système global interconnecté (concept $\mathrm{M}^{2} \mathrm{M}^{3}$ ). Un produit vendu sera personnalisable. Il pourra communiquer avec les machines dans sa phase de réalisation avec des étiquettes électroniques. Cette rupture organisationnelle consistera à façonner des unités de production autonomes et intelligentes. Les capacités de décision et l'intelligence des processus de production seront déportées.

Nous vivons une expansion des capacités technologiques depuis 20 ans. Ce constat n'est pas sans impact sur les enseignements scientifiques et technologiques que nous devons apporter auprès de nos étudiants (expertise \& compétences : Fig. 2). Ces évolutions technologiques ont été guidées d'une part, par la volonté de suivre l'évolution des technologies et des sciences (vitrine technologique) et d'autre part, par la volonté de collaborer avec le tissu industriel de notre région. Les grands sauts technologiques ont été réalisés en corrélation directe avec les

\footnotetext{
${ }^{3} \mathrm{M} 2 \mathrm{M}$ : Machine to Machine
} 
projets tuteurés que nous menons avec les étudiants depuis la création de notre Institut Universitaire de Technologie dans l'Indre. Depuis quelques années, nos enseignements dans différentes écoles d'ingénieurs (HEI Campus Centre, Polytech'Orléans, INSA Centre val de Loire) et nos travaux de recherche ont également contribué à cette évolution (Laboratoire PRISME ${ }^{4}$, Pôle IRAus ${ }^{5}$ ). Dans la suite de cet article, nous proposons au lecteur un exemple de solutions technologiques utilisées avec les étudiants afin de les conduire vers ce concept de l'industrie 4.0 .

\section{STRUCTURE PYRAMIDALE D'UNE ENTREPRISE EN LIAISON AVEC DES BESOINS EN INFORMATIQUE INDUSTRIELLE}

Dans le monde exigeant du contrôle-commande de processus, l'accès à des données de processus et/ou d'usine en ligne et en temps "souhaité" est crucial. La croissance d'une entreprise se traduit par une croissance parallèle du volume des données issues du processus et de la demande de traduction en informations pertinentes pour les équipes d'exploitation et de direction [3]. Souvent, les personnes qui ont en charge un système d'information doivent s'attacher à connecter et récupérer les informations pertinentes de leur usine via leur système informatique [4]. Lorsque cela fonctionne, les personnes concernées ne peuvent souvent pas utiliser efficacement et rapidement les données brutes issues de la production pourtant essentielles pour le contrôle des coûts d'exploitation (Fig. 3(a)).
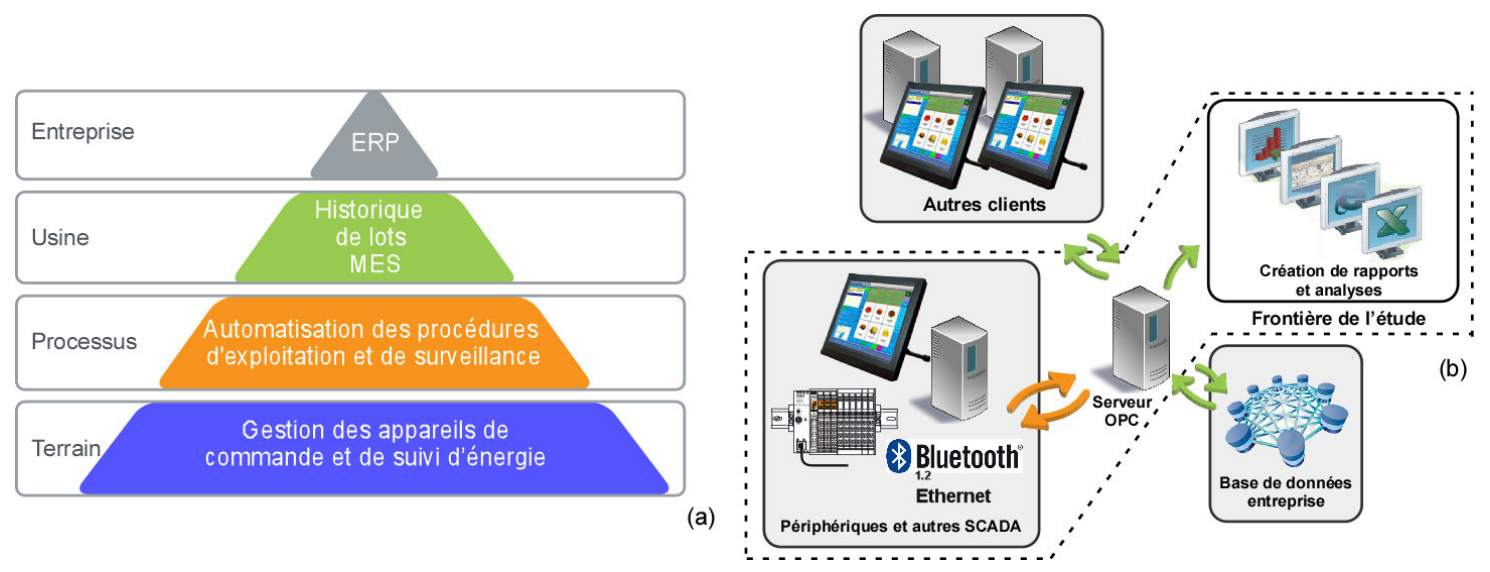

Fig. 3 : Architecture pyramidale dans le Concept $\mathrm{CIM}^{6}$

Le travail présenté consiste à mettre en place une structure opérationnelle montrant les fondements d'une architecture industrielle communicante (Fig. 3). Il apporte un grand nombre de compléments d'information par rapport aux articles [5], [6], [7]. A partir des informations émanant du processus, on souhaite développer et utiliser plusieurs clients dans une démarche

\footnotetext{
${ }^{4}$ PRISME : Pluridisciplinaire de Recherche en Ingénierie des Systèmes, Mécanique et Energétique

${ }^{5}$ IRAus : Images, Robotiques, Automatique et Signal

${ }^{6} \mathrm{CIM}$ : Computer Integrated Manufacturing
} 
$\mathrm{SCADA}^{7}[8]$. Nous montrons dans cet article plusieurs solutions à implémenter pour effectuer un large panel de tests. Pour cela, les données sont centralisées dans un serveur OPC et mises à disposition vers différents clients OPC, l'ensemble permettant de suivre "en ligne", un certain nombre de contenus de variables $\left(\right.$ Items $\left.^{8}\right)$. Les choix proposés feront également l'objet d'une réflexion à mener avec les étudiants sur les points suivants : stabilité de Windows, du pack Office, robustesse de la solution, sécurité... Une consultation des variables avec des objets portables (tablette et Smartphone) est également possible et présentée.

\subsection{Détail de l'application dans une démarche SCADA}

L'environnement architectural de travail est présenté Fig. 4. Il reprend le concept présenté Fig. 3(b). Le processus est piloté par une partie commande sur laquelle est implantée une application d'automatisme répondant par exemple à une recette de fabrication. Un certain nombre de logiciels doivent être installés sur l'ordinateur qui sera relié au réseau $\operatorname{LAN}^{9} \mathrm{du}$ département de formation (Fig. 4). Les solutions hardware utilisées s'insèrent dans un contexte où les communications numériques sans-fil étendent leur influence [9].

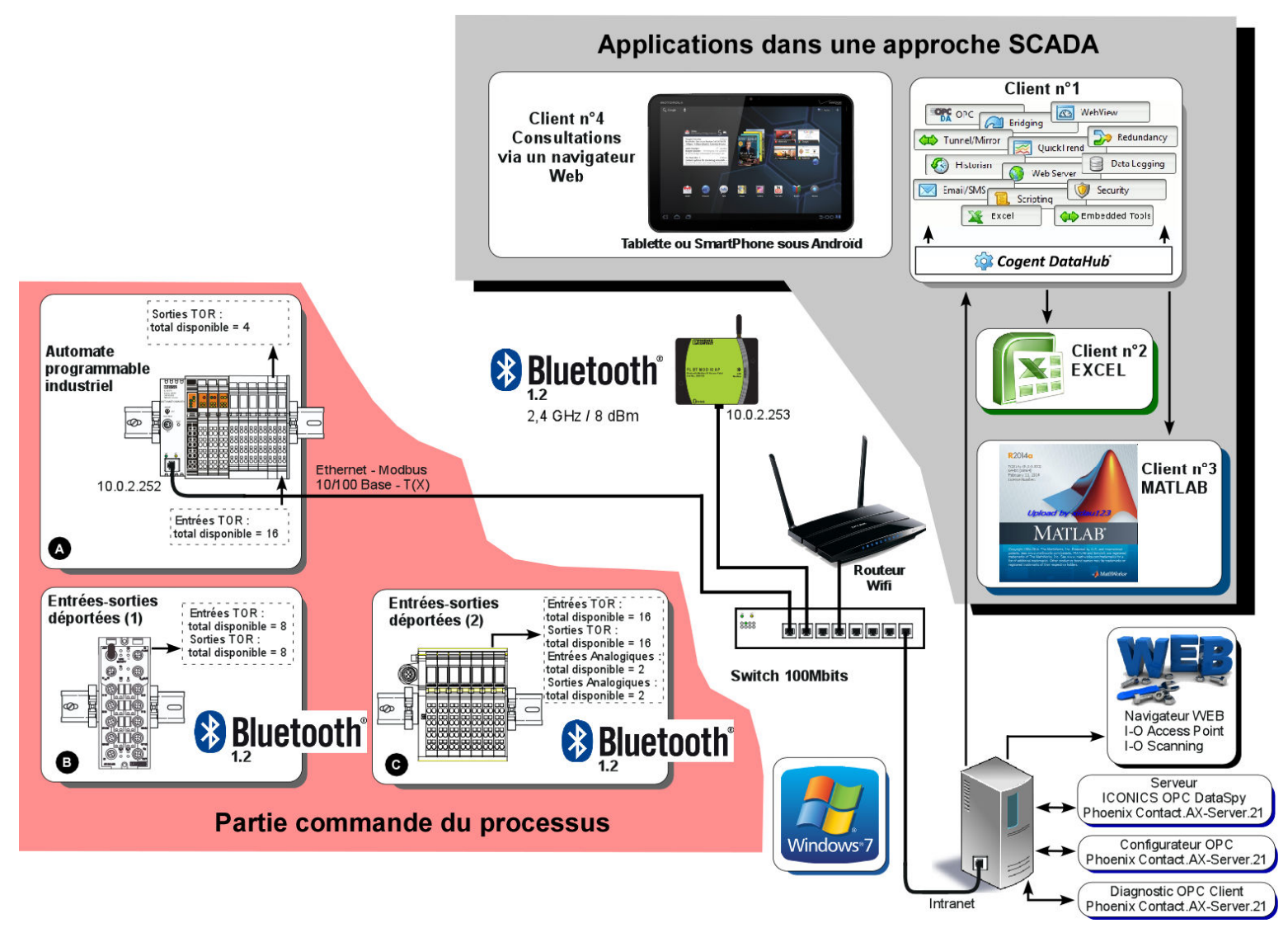

Fig. 4 : Architecture de l'installation mise en ouvre

\footnotetext{
${ }^{7}$ SCADA : Supervisory Control and Data Acquisition. L'objectif d'un environnement SCADA est de mener une conduite réactive d'un processus. Un environnement SCADA comprend 3 sous-ensembles : la fonction commande, la fonction surveillance et la fonction supervision.

${ }^{8}$ Item : désignation d'un objet associé à une variable à déclarer permettant d'être traitée par le serveur OPC et les clients potentiels.

${ }^{9}$ LAN : Local Area Network
} 
Une antenne omnidirectionnelle Bluetooth permet un acheminement bidirectionnel des variables hébergées par l'API au temps de cycle de $25 \mathrm{~ms}$ dans la pire des configurations (c'està-dire 7 modules Wireless-IO). La bande de fréquence utilisée peut donner lieu à l'apparition d'interférences provenant d'autres appareils. Il est donc nécessaire d'employer un procédé de transmission robuste. Le constructeur utilise le principe du saut de fréquence (FHSS ${ }^{10}$ ) [3]. En l'occurrence, la bande de fréquence disponible de 2,402 à 2,480 GHz est répartie sur 79 canaux avec une largeur de bande de $1 \mathrm{MHz}$ chacun. Le canal de transmission est changé 1600 fois par seconde (toutes les $625 \mu \mathrm{s}$ ). Si des interférences surviennent par le biais d'éventuels autres appareils radio, le télégramme est alors répété par la suite, sur un nouveau canal. Dans la plupart des cas, le correcteur d'erreurs intégré FEC $^{11}$ est en mesure d'identifier les erreurs et de les corriger automatiquement. Le principe du saut de fréquence contribue essentiellement à la protection des datagrammes contre les écoutes, puisque le changement de fréquence s'effectue de manière pseudo aléatoire. Un cryptage des données à 128 bits garantit par ailleurs une grande sécurité.

\subsubsection{Verrous logiciels}

Différents serveurs OPC peuvent être déployés et utilisés afin de répondre aux exigences du sujet présenté dans cet article (CODRA Composer OPCServer, Matrikon OPC, Resolvica OPC, SV OPC...). Ces serveurs font l'objet de comparatifs dans différentes revues consacrées à l'informatique industrielle depuis plusieurs années. Notre choix s'est porté sur une solution proposée par Iconics [10], société internationale qui commercialise des environnements de développement répondant aux problématiques de la Fig. 3 avec différents "packages". Cette solution fonctionnera en partenariat avec le serveur PhoenixContact.AX-Server.21 afin d'associer l'ensemble des Items. Pour pouvoir lier les différents clients au serveur OPC, il faut également installer le logiciel Cogent DataHub [11]. Cogent DataHub permettra d'associer via le serveur OPC Iconics, les clients $n^{\circ} 2,3$ et 4 (Fig. 4). Le client $n^{\circ} 2$ utilise un logiciel du pack Office. Différents essais relatifs à l'application ont été validés avec des versions différentes (XP-Pro, 2010-Pro et 2013-Pro). Cependant, il faut apporter une attention particulière pour les versions 32 bits ou 64bits. Le client $\mathrm{n}^{\circ} 3$ utilise la version de Matlab R2014a avec la toolbox OPC. Le client $n^{\circ} 4$ supporte le système d'exploitation Androïd. C'est une tablette de type grand public. La communication avec les différents services hébergés dans l'ordinateur relié au réseau s'effectue à partir du routeur Wifi qui aura été préalablement configuré (Fig. 4).

\subsubsection{Création de l'application concernant la partie commande hébergée dans l'automate programmable industriel}

L'application gérant le processus est développée avec l'environnement de programmation PCWORX 6.0 (IEC 61131-3). Cette application n'a pas vocation à gérer un fonctionnement complexe. Néanmoins, elle doit permettre la gestion globale de la communication Bluetooth ainsi que la gestion de l'ensemble des entrées-sorties physiques pour notre sujet (Fig. 5). La Photo 1 présente les 3 coffrets concernés de la partie commande (A, B et C).

\footnotetext{
${ }^{10}$ FHSS : Frequency Hopping Spread Spectrum

${ }^{11}$ FEC : Forward Error Correction
} 


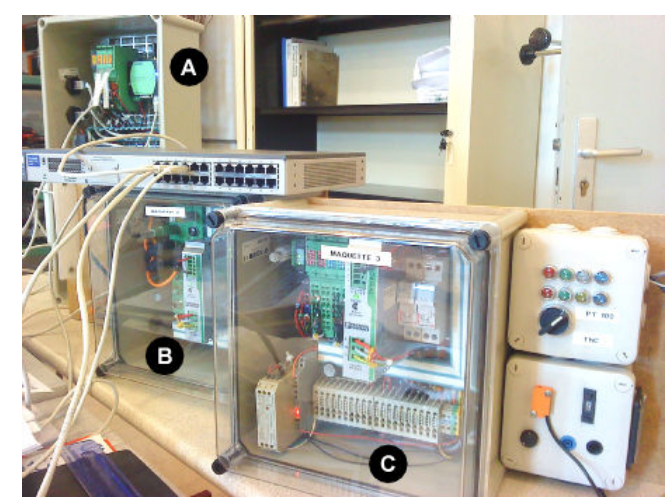

Photo 1 : Photo de l'installation

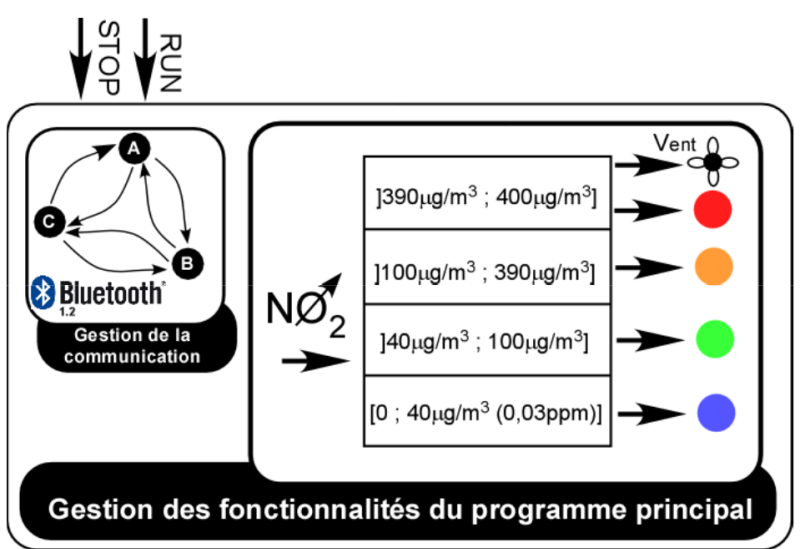

Fig. 5 : Fonctions à gérer

Les fonctionnalités des coffrets $\mathrm{A}, \mathrm{B}$ et $\mathrm{C}$ sont précisées Fig. 4. La Fig. 6 présente la configuration matérielle Bluetooth hébergée dans le coffret $\mathrm{C}$.
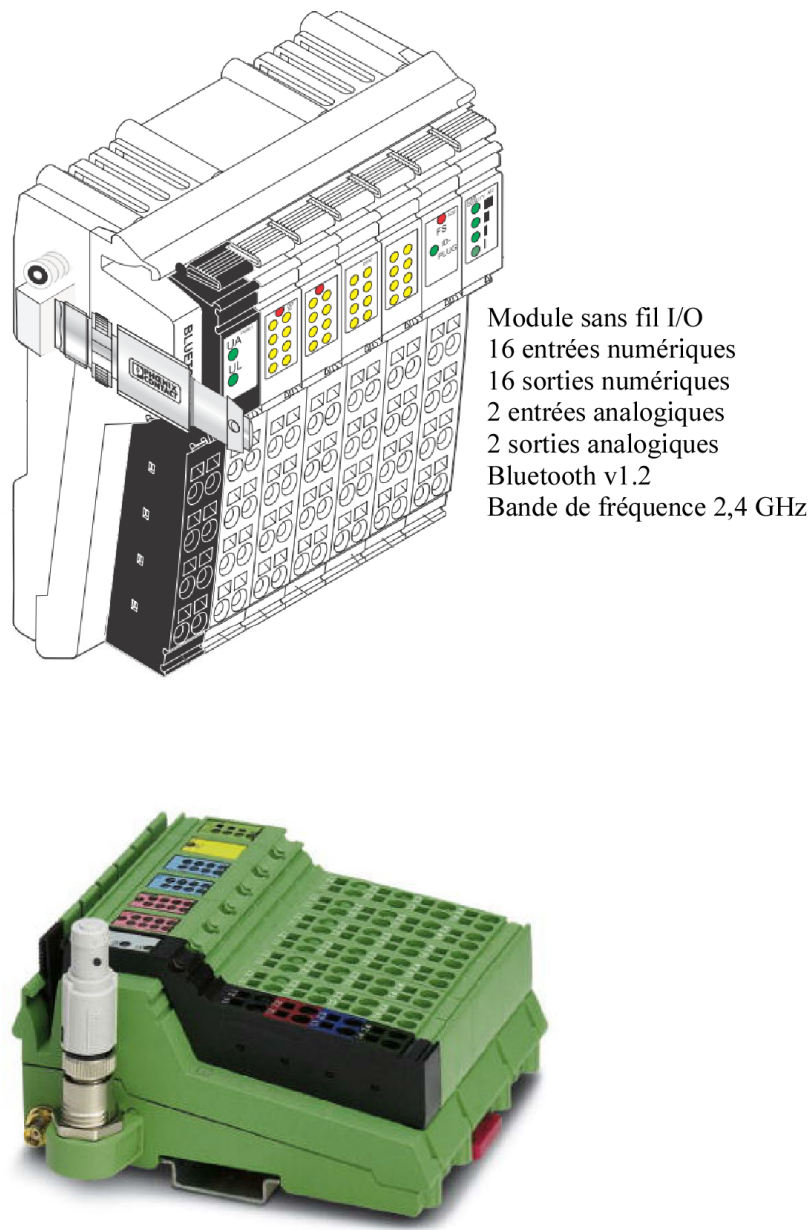

Fig. 6 : Module Bluetooth dans le coffret $\mathrm{C}$

Le sous-programme concernant la gestion de la communication n'est pas présenté dans cet article. C'est une fonction particulière qui dépend d'un sous-programme associé à des fonctions spéciales dépendantes de la bibliothèque de communication. Les fonctionnalités du programme principal reprennent les contraintes de base concernant la surveillance du taux de dioxyde 
d'azote $\left(\mathrm{NO}_{2}\right)$ dans un tunnel routier [12]. Un capteur $\mathrm{NO}_{2}$ renseigne une entrée de type analogique $[0,10 \mathrm{~V}]$. Le capteur $\mathrm{NO}_{2}$ a les caractéristiques suivantes : 0 à $500 \mu \mathrm{g} / \mathrm{m}^{3}(0-$ $0,375 \mathrm{ppm})$ pour une plage de mesure de $[0-10 \mathrm{~V}]$.

Des sorties de signalisation et une ventilation forcée du tunnel sont associées à des seuils de contrôle (Fig. 7).

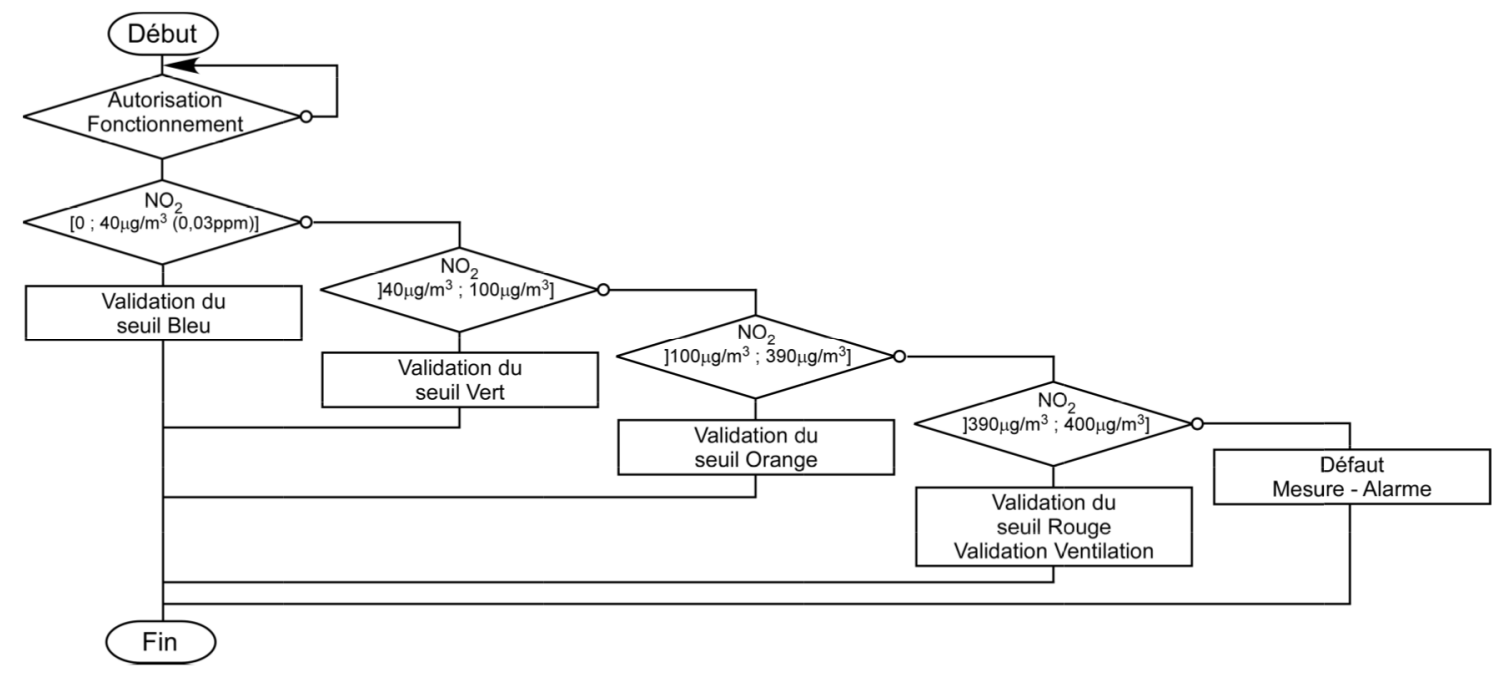

Fig. 7 : Organigramme de fonctionnement du programme principal

Un travail complémentaire sur différents aspects techniques peut également être mené avec les étudiants : résolution de la carte des entrées analogiques, résolution, traitements numériques (INT, Word, Float...), mise à l'échelle.

Le développement de l'application hébergée dans l'automate doit respecter les contraintes exprimées dans l'organigramme de la Fig. 7 tout en associant, les variables à traiter (variables qui seront associées individuellement à un Item dans le service OPC (Tableau 1)).

Tableau 1 : Déclaration des entrées/sorties et des items

\begin{tabular}{|c|c|c|c|c|c|c|c|}
\hline Nom & Description & $\begin{array}{l}\text { Entrées } \\
\text { Mnémonique }\end{array}$ & Item & Localisation & Type & Info initiale & Détail \\
\hline Demande d'arrêt & Bouton poussoir : demande d'arrêt & STOP & Demande_Arret & A & TOR & $1(\mathrm{NF})$ & Bit \\
\hline $\begin{array}{l}\text { Autorisation de } \\
\text { fonctionner de } \\
\text { l'installation }\end{array}$ & Bouton poussoir : autorisation de fonctionner & RUN & Demande_Marche & B & TOR & o(NO) & Bit \\
\hline $\begin{array}{l}\text { Valeur du taux de } \\
\text { NO2/m3 }\end{array}$ & Capteur : taux de concentration de $\mathrm{NO} 2 / \mathrm{m} 3$ & Valeur_Analogique_Entree2_INT & Valeur_Analogique_Entree2_INT & & Ana & 0 & Mot de 16 Bits \\
\hline \multicolumn{8}{|c|}{ Sorties } \\
\hline Nom & Description & Mnémonique & Item & Localisation & Type & Info initiale & Détail \\
\hline $\begin{array}{l}\text { Seuil de NO2: } \\
\text { signalisation bleue }\end{array}$ & $\begin{array}{l}\text { Voyant de signalisation seuil } \mathrm{NO} 2 \text { compris entre }[0 ; 10 \mathrm{mg} / \mathrm{m} 3 \\
(0,03 \mathrm{ppm})]\end{array}$ & Seuil_Bleu & Seuil_Bleu & & TOR & 0 & Bit \\
\hline $\begin{array}{l}\text { Seuil de NO2: } \\
\text { signalisation verte }\end{array}$ & $\begin{array}{l}\text { Voyant de signalisation seuil NO2 compris entre }] 40 \mathrm{mg} / \mathrm{m} 3 \\
\qquad 100 \mathrm{mg} / \mathrm{m} 3]\end{array}$ & Seuil_Vert & Seuil_Vert & & TOR & 0 & Bit \\
\hline $\begin{array}{l}\text { Seuil de NO2: } \\
\text { signalisation jaune }\end{array}$ & $\begin{array}{l}\text { Voyant de signalisation seuil NO2 compris entre }] 100 \mathrm{mg} / \mathrm{m} 3 \\
\qquad 390 \mathrm{mg} / \mathrm{m} 3]\end{array}$ & Seuil_Jaune & Seuil_Jaune & & TOR & 0 & Bit \\
\hline $\begin{array}{l}\text { Seuil de } \mathrm{NO} 2 \text { : } \\
\text { signalisation rouge }\end{array}$ & Voyant de signalisation seuil $\mathrm{NO} 2>390 \mathrm{mg} / \mathrm{m} 3$ & Seuil_Rouge & Seuil_Rouge & C & TOR & 0 & Bit \\
\hline $\begin{array}{l}\text { Motorisation } \\
\text { ventillation forcée }\end{array}$ & Ventillation forcée du unnel si la valeur NO2 $>390 \mathrm{mg} / \mathrm{m} 3$ & Ventilation & Ventilation & & TOR & 0 & Bit \\
\hline
\end{tabular}




\subsection{Validation de l'état de fonctionnement des entrées-sorties à partir d'un navigateur Internet}

La passerelle Bluetooth (adresse IP : 10.0.2.253) permet d'effectuer un contrôle dynamique des entrées-sorties (IO Scanning). Cette fonction sécurisée (appelée également "monitoring") permet de contrôler le contenu de chaque entrée ou sortie physique du processus. Cette fonction n'est pas nouvelle, mais son accès sécurisé permet d'aborder l'utilisation et les recommandations concernant l'usage des mots de passe (Fig. 8 (a)).

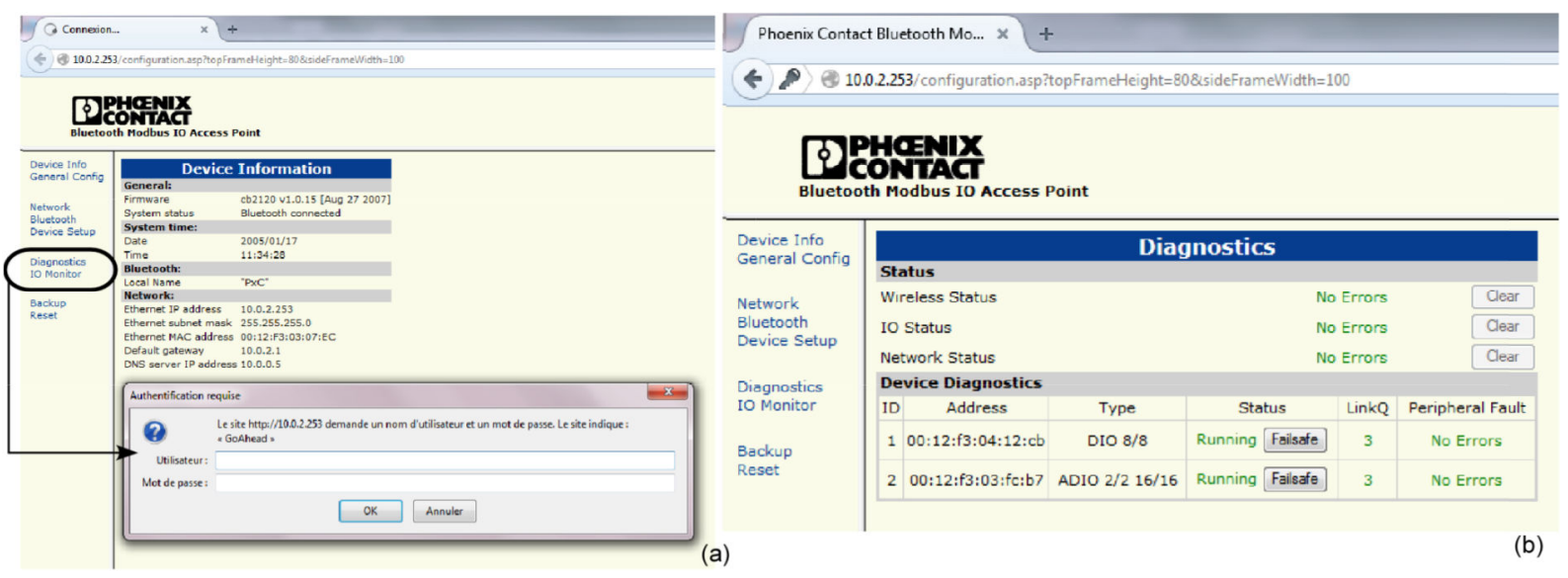

Fig. 8 : Diagnostic et IO Scanning (gestion sécurisée avec mot de passe)

L'utilisation de mots de passe "forts" est l'une des briques de base dans la sécurisation d'un système d'information nécessitant la mise en place d'une réelle politique de sécurité. Malheureusement, cette première étape est souvent absente. Il est par conséquent, assez fréquent de trouver des comptes avec des mots de passe triviaux, sans mot de passe ou avec des mots de passe par défaut [13]. Un bon mot de passe (mot de passe dit "fort"), sera donc difficile à retrouver même à l'aide d'outils automatisés, tout en restant facile à retenir. En effet, si un mot de passe est trop compliqué à retenir, l'utilisateur mettra en place des moyens mettant en danger la sécurité du système d'information, comme par exemple l'inscription du mot de passe sur un papier collé sur l'écran ou sous le clavier. Pour ce faire, il existe des moyens mnémotechniques pour fabriquer et retenir des mots de passe "forts".

$\checkmark$ Méthode phonétique :

Cette méthode consiste à utiliser les sons de chaque syllabe d'une phrase facile à retenir. Par exemple la phrase "J'ai acheté huit cd pour cent euros cet après-midi" deviendra "ght8CD\%E7am".

$\checkmark$ Méthode des premières lettres :

Cette méthode consiste à garder les premières lettres d'une phrase (citation, paroles de chanson...) en veillant à ne pas utiliser que des minuscules. Par exemple, la citation "un tiens vaut mieux que deux tu l'auras" donnera "1tvmQ2tlA".

Cette partie du travail permet également d'aborder un autre point très important : la réglementation 21 CFR partie 11, qui définit les critères selon lesquels les dossiers et signatures électroniques seront considérés comme équivalents à des dossiers sur support papier et des signatures manuscrites [14]. L'accès étant validé, il est possible de contrôler les différents statuts de la configuration (Fig. 8 (b)) ainsi que l'ensemble des entrées/sorties associées à leur état ou leur contenu (Fig. 9). 

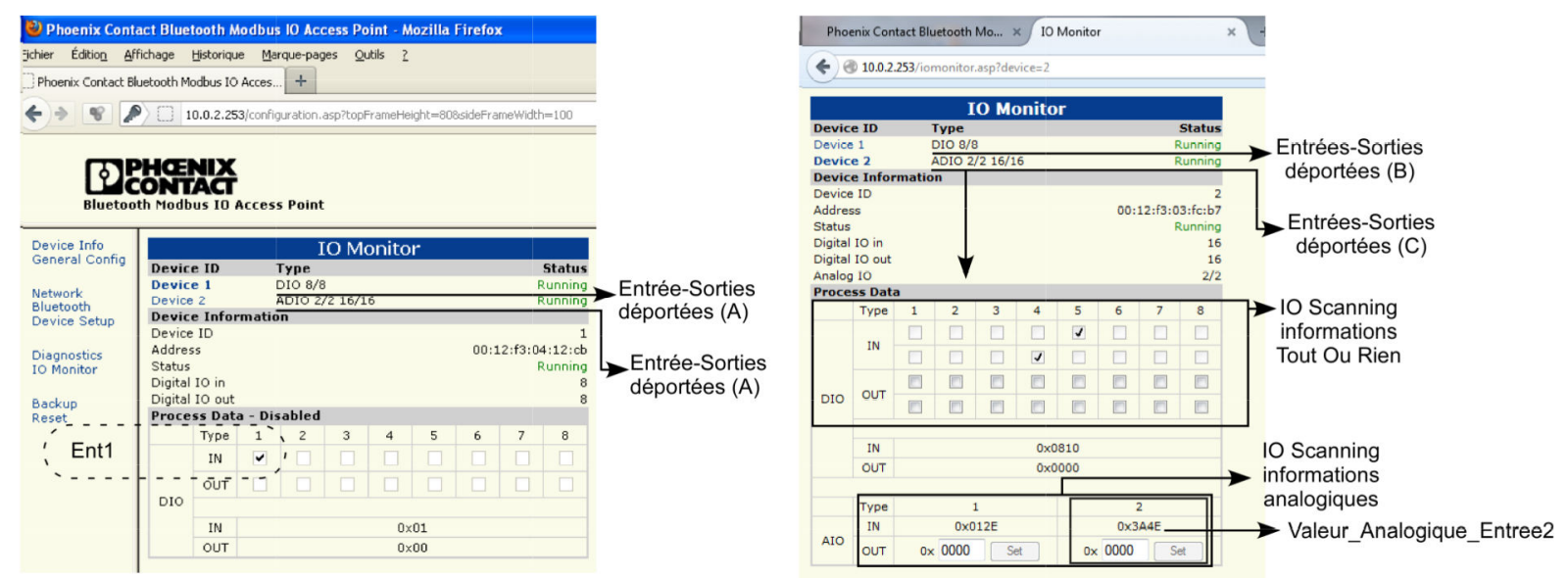

Fig. 9 : Fonction IO Scanning $(A, B, C)$

\section{DEVELOPPEMENT ET UTILISATION DES DIFFERENTS CLIENTS}

L'objectif de cet article n'est pas de présenter l'ensemble des résultats issus de nos différents tests. Les résultats significatifs pour les quatre clients sont présentés ci-dessous. Ils apportent de nombreux éclaircissements sur les outils disponibles sur "étagère" et ce que nous pouvons en faire pédagogiquement avec nos étudiants. Pour permettre un lien dynamique entre les informations émanant du terrain (Entrées-Sorties, ...) et la solution de "reporting" avec Excel, il faut au préalable s'affranchir de plusieurs couches logicielles associées à des paramètres. La première couche consiste à lancer le serveur OPC adéquat. L'ordinateur utilisé dans le cas présent, dispose de plusieurs serveurs installés dans un environnement de contrôle et de configuration : ICONICS OPC DataSpy. Dans notre cas, nous avons utilisé le serveur préconisé par le constructeur : PhoenixContact.AX-Server.21 (Fig. 10). Ce serveur doit être actif pour l'ensemble des différents essais.

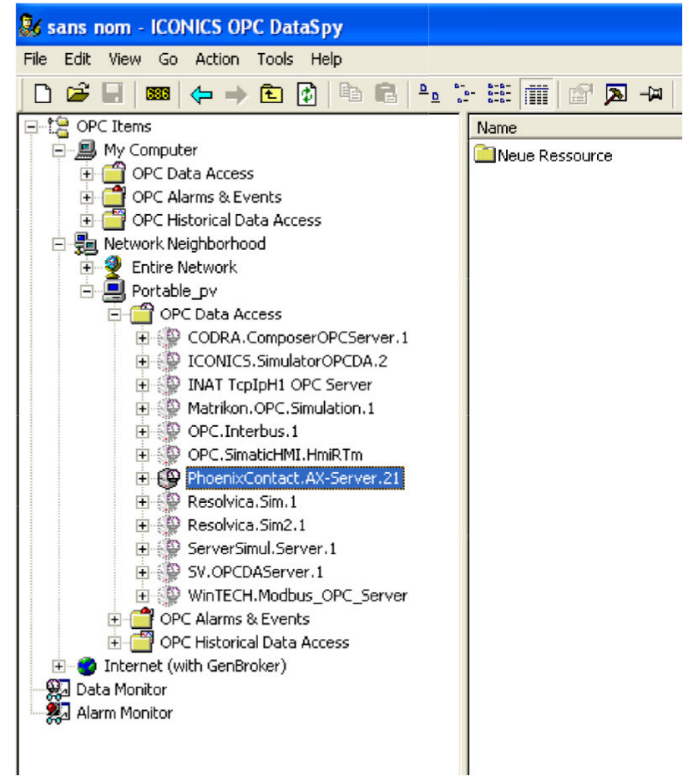

Fig. 10 : Activation du serveur OPC

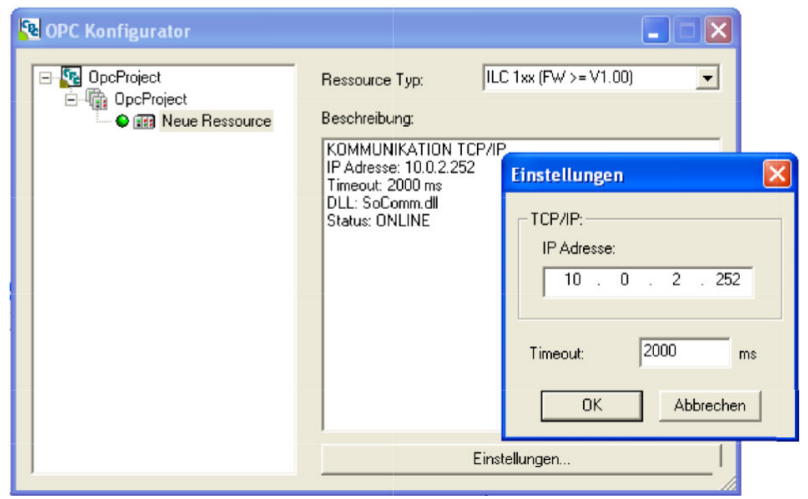

Fig. 11 : Déclaration de l'adresse IP de l'automate pour le serveur OPC 
Après activation du serveur, nous devons dans un autre environnement logiciel, déclarer "la porte" d'entrée et de sortie des contenus des Items qui vont être exploités par la suite. Dans notre situation, c'est l'adresse IP de l'API associée à sa référence constructeur ILC1xx(FW $>=$ V1.00), (Fig. 11).

\subsection{Le client $n^{\circ} 1$}

Aujourd'hui, OPC est devenu le service de choix dans un environnement de contrôlecommande de processus. Comme nous l'avons déjà présenté, il existe un grand nombre de serveurs OPC permettant de disposer d'une connectivité avec différents automates programmables industriels, PC industriels... Très souvent, les fabricants de matériel d'automation proposent une suite logicielle OPC compatible avec leur gamme de produits. Cela permet ensuite aux éditeurs de logiciels de supervision de créer des applications "client OPC" pour accéder facilement à des données en temps réel provenant d'un processus de fabrication, d'un système, d'une machine... L'éditeur Cogent Real-Times Systems [11] propose une suite logicielle permettant de disposer de très nombreux services (Fig. 12).

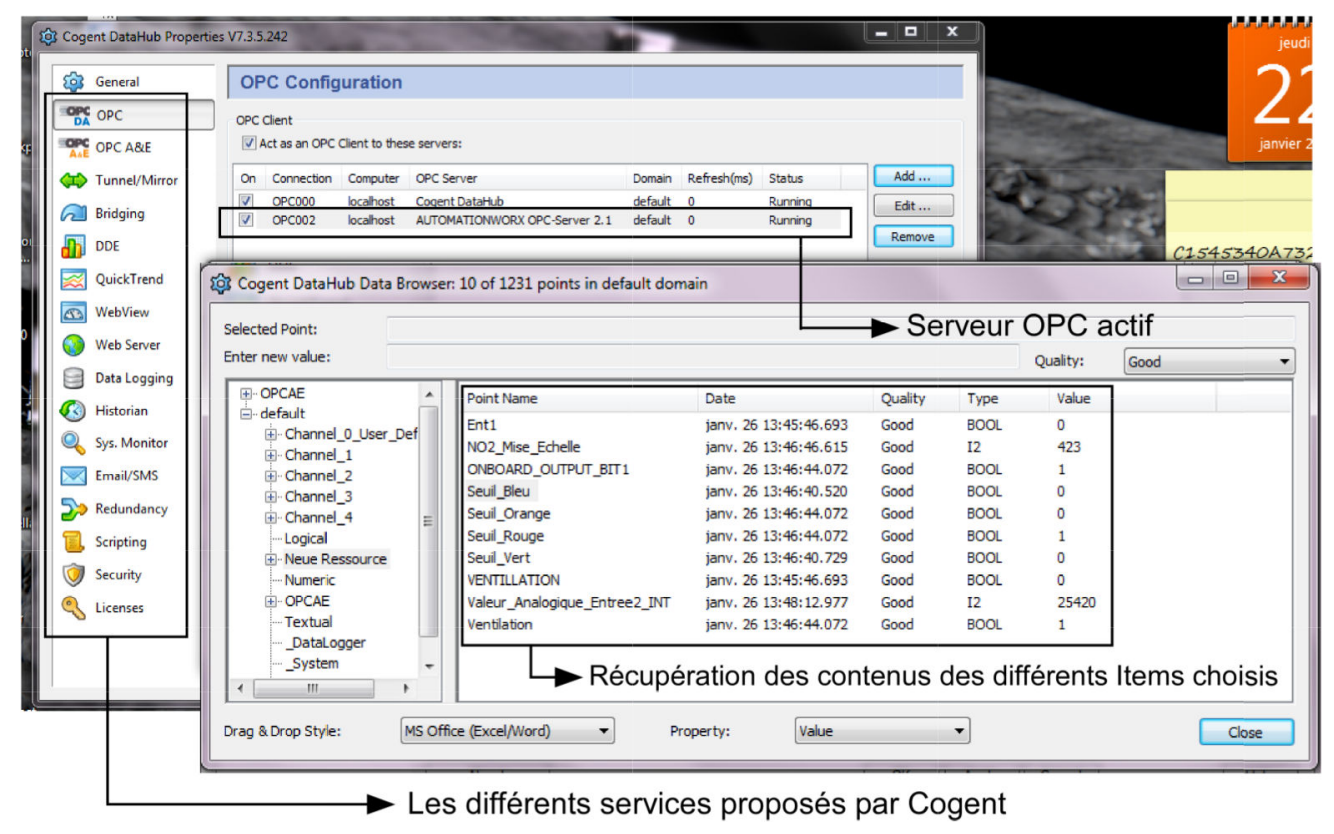

Fig. 12 : Connexion au serveur OPC PhoenixContact.AX-Server.21

L'environnement logiciel permet la connexion au serveur OPC actif. Le contenu des différents Items est récupérable de manière transparente. Les informations peuvent être archivées à la demande. La Fig. 13 montre un traitement possible concernant la mesure du taux de concentration du $\mathrm{NO}_{2} / \mathrm{m}^{3}$.

Le programme hébergé dans l'automate tient compte des contraintes associées à la Fig. 7. Les seuils d'alarmes en fonction de la valeur du taux de $\mathrm{NO}_{2}$ sont donc traités et peuvent être affichés également avec la fonction QuickTrend (Fig. 14). 


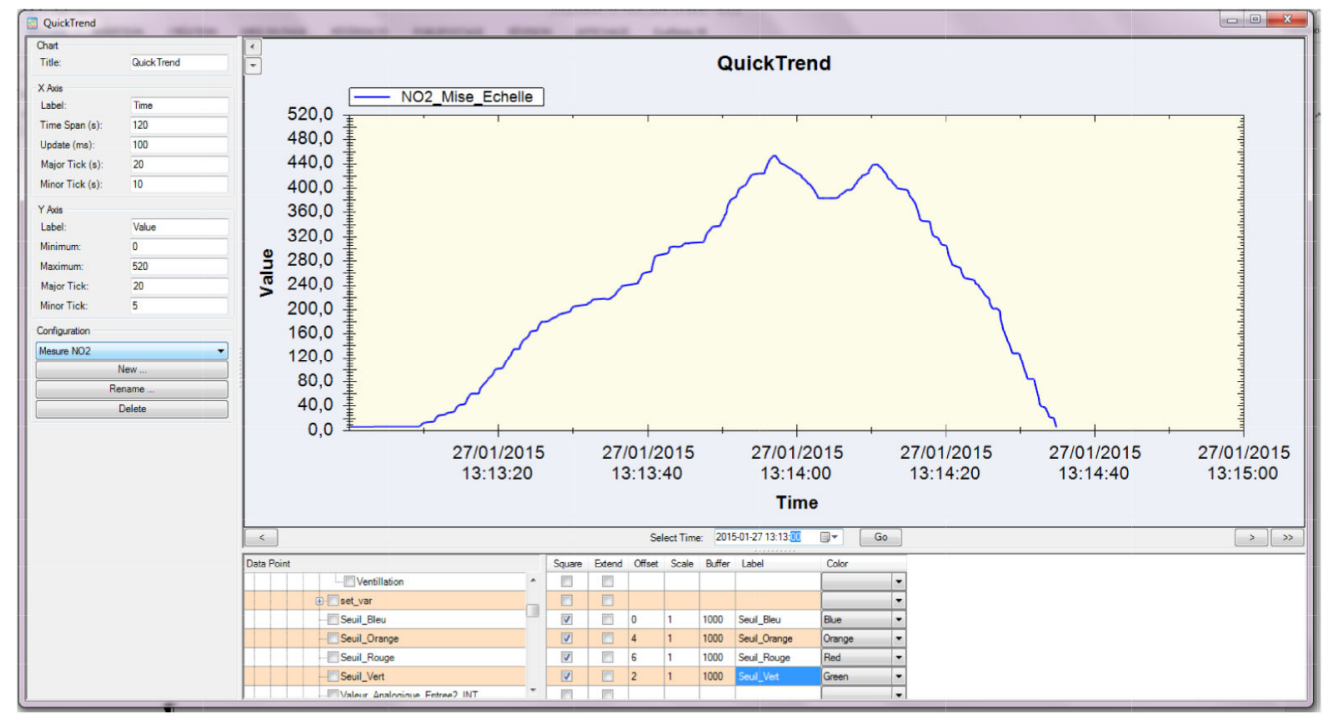

Fig. 13 : Enregistrement au fil de l'eau du taux de concentration $\mathrm{du}^{\mathrm{NO}_{2}} / \mathrm{m}^{3}$ avec la fonction QuickTrend sur la campagne de mesures

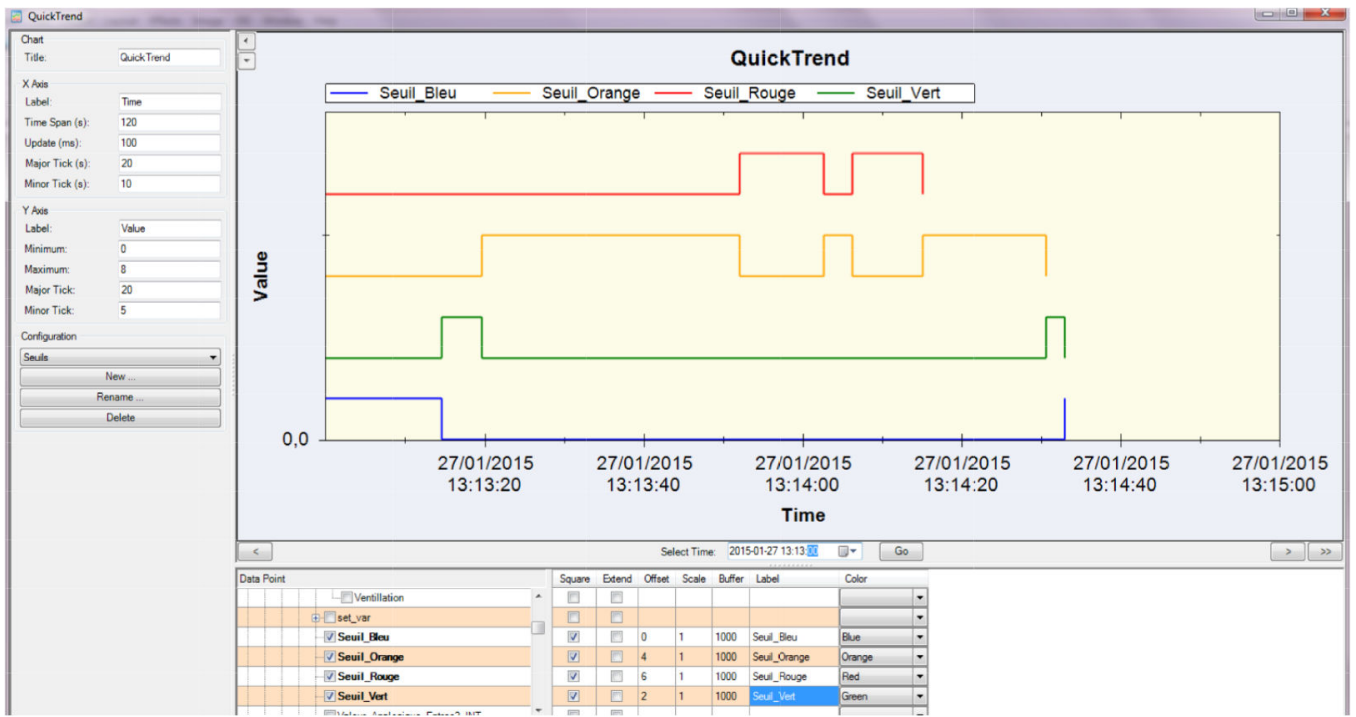

Fig. 14 : Enregistrement au fil de l'eau des seuils d'alarmes avec la fonction QuickTrend

\subsection{Le client $n^{\circ} 2$}

Pour permettre un lien dynamique entre les informations émanant du terrain (EntréesSorties...) et la solution de "reporting" avec Excel, il faut au préalable s'affranchir de plusieurs couches logicielles associées à des paramètres. La première couche consiste à lancer le serveur OPC adéquat (dans notre cas : PhoenixCon-tact.AX-Server.21 (Fig. 10)). A partir de cette phase, Cogent DataHub dispose de la capacité à effectuer une fonction de drag \& drop d'Item vers une cellule choisie dans une feuille Excel. Une Macro complémentaire en Visual Basic et spécifique a été développée sous Excel pour aboutir aux résultats complets présentés Fig. 16 :

- la répartition des seuils sur la campagne de mesures,

- l'évolution du taux de $\mathrm{NO}_{2}$ en fonction du temps. 
Ce travail complémentaire peut être effectué avec les étudiants. Il soulève un point très important dans l'acquisition et le stockage de grandeurs numériques associés à la fréquence d'échantillonnage d'une mesure. Dans notre cas (phénomène lent), pour que l'essai soit rapide et concluant, nous avons réglé cette valeur à 3 secondes dans la macro concernée. La Fig. 15 présente les différentes tâches qui doivent être gérées (1) : programme développé dans l'environnement de développement pour la programmation de l'automate ; 2 : récupération des différents Items pouvant être utilisés sous l'environnement Cogent DataHub ; 3 : client Excel et traitement des Items dans l'application d'interface Homme-machine en cours de développement).

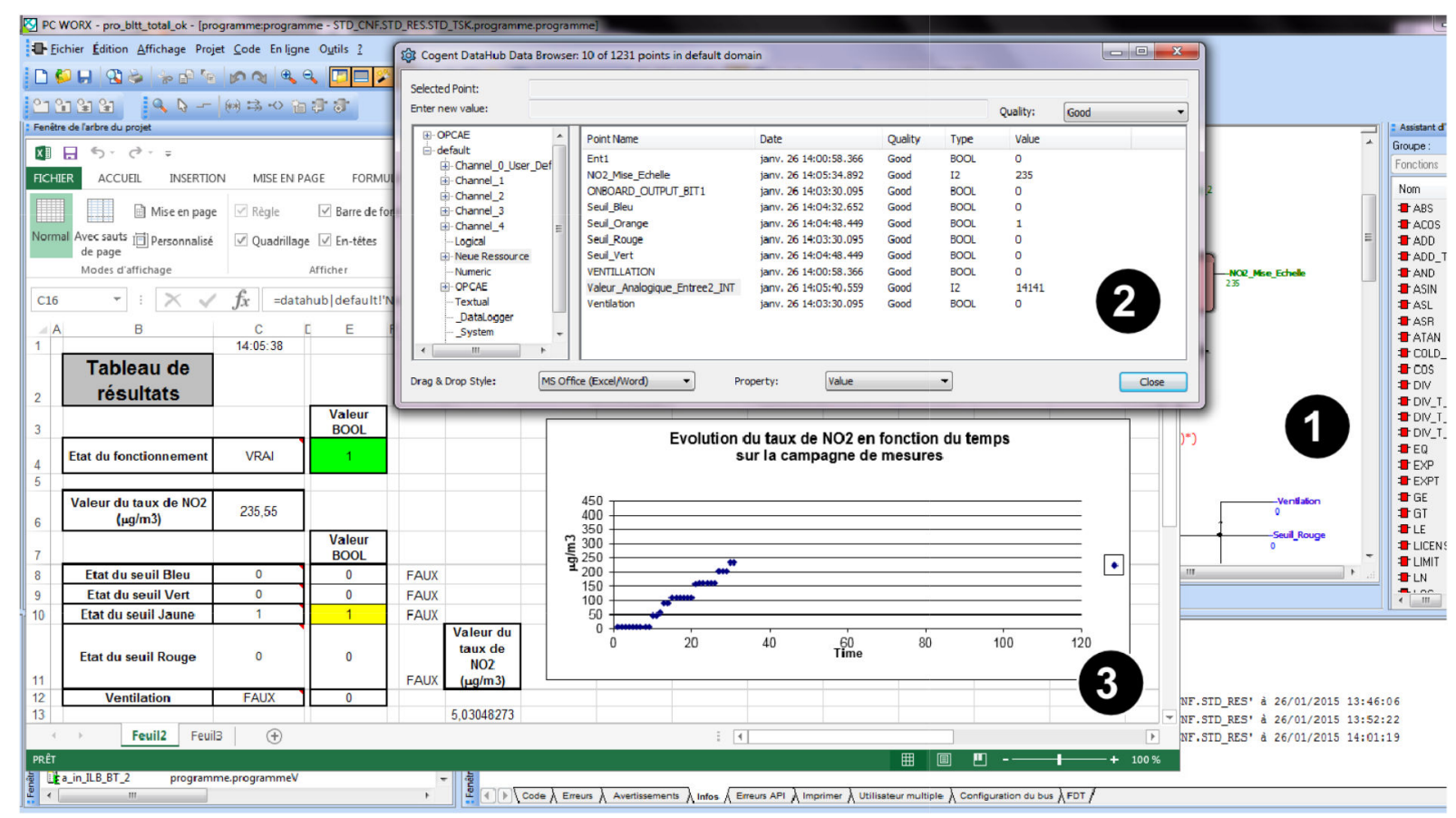

Fig. 15 : Différentes tâches à traiter

\subsubsection{Les résultats significatifs pour le client $n^{\circ} 2$}

L'objectif de ce paragraphe n'est pas de développer l'ensemble des tests effectués mais de présenter des résultats significatifs (Fig. 16). Cette partie de travail montre que l'environnement Excel est utilisé pour répondre à un besoin de "reporting" d'informations, en liaison directe avec le processus. Ce travail permet de soulever énormément de préjugés de la part des étudiants vis-à-vis de son usage classique du quotidien en utilisant des fonctions de type : Mise en Forme Conditionnelle, =SI(C8=VRAI;"OK";"FAUX")...

Le suivi "au fil de l'eau" du taux de $\mathrm{NO}_{2}$ dans le tunnel associé aux différents seuils et à l'alarme (ventilation forcée) permet en parallèle de pouvoir effectuer des traitements classiques avec différentes représentations : histogramme, camembert... 


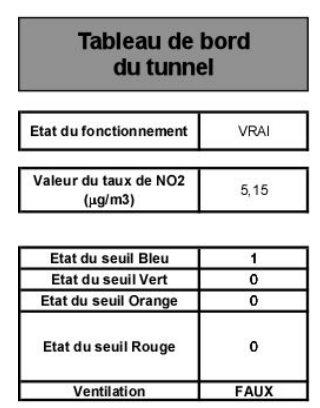

Recopie de l'entrée analogique :

\begin{tabular}{|c|c|c|c|}
\hline Répartion : seuil Bleu & $22,2 \%$ & $\begin{array}{c}\text { Nb occurrence } \\
\text { seuil Bleu }\end{array}$ & \\
\hline Répartition : seuil Vert & $22,2 \%$ & $\begin{array}{c}\begin{array}{c}\text { Nb occurrence } \\
\text { seuil Vert }\end{array} \\
\text { sets }\end{array}$ & 2 \\
\hline Répartition : seuil Orange & $33,3 \%$ & Nb occurrence & 3 \\
\hline Répartition : seuil Rouge & $22,2 \%$ & $\begin{array}{l}\begin{array}{c}\text { Nb occurrence } \\
\text { sevilupouge }\end{array} \\
\end{array}$ & 2 \\
\hline
\end{tabular}

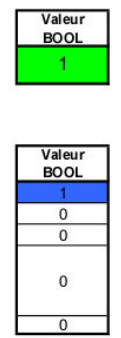

Evolution du taux de NO2 en fonction du temps sur la campagne de mesures

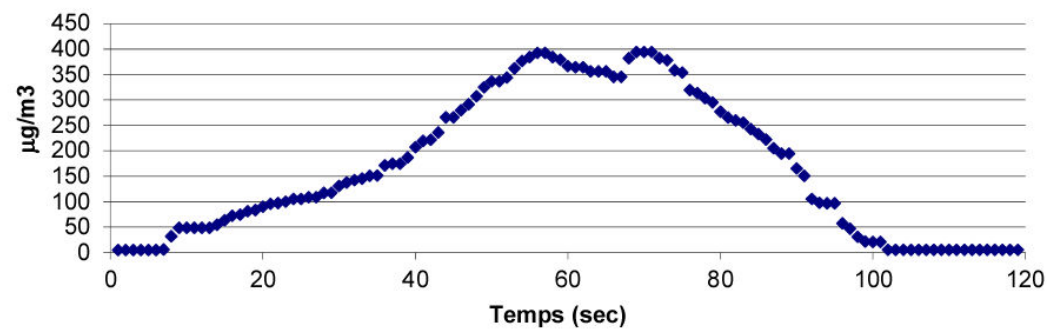

Répartition des seuils sur la campagne de mesures

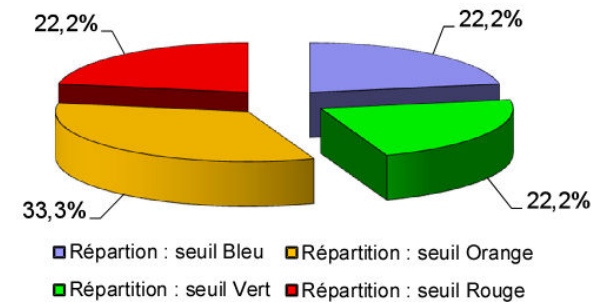

口Répartition : seuil Vert aRépartition : seuil Rouge

Fig. 16 : Résultats concernant le suivi du taux de $\mathrm{NO}_{2}$

\subsection{Le client $n^{\circ} 3$}

La Toolbox OPC de Matlab (R2014a) permet de fournir une connexion aux serveurs OPC $\mathrm{DA}^{12}$ et OPC HDA ${ }^{13}$. Cette connexion permet de lire, écrire et enregistrer des contenus d'Items à partir de différents périphériques tels que, les systèmes de contrôle-commande distribués, les systèmes de surveillance et d'acquisition de données... Le client $\mathrm{n}^{\circ} 3$ peut donc récupérer des informations issues de notre application. Il pourra également par la suite et si besoin, effectuer de nombreux traitements algorithmiques spécifiques déjà implémentés. La Fig. 17 présente la configuration à adopter.

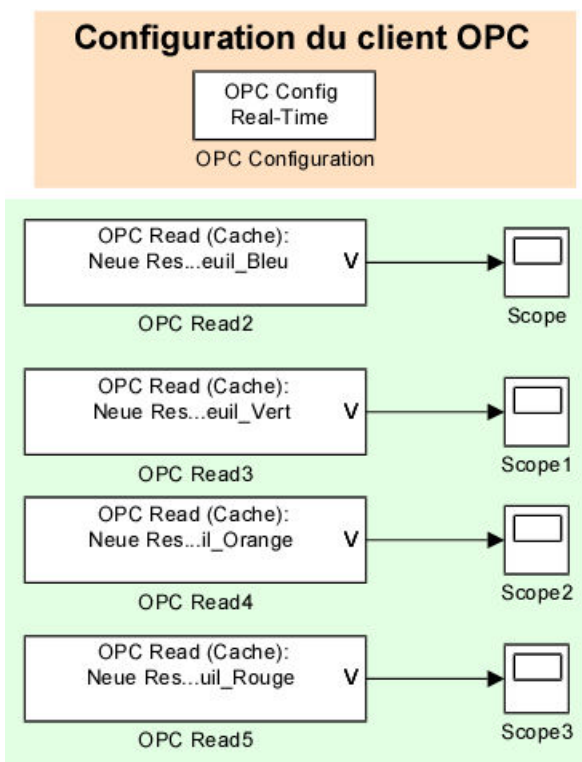

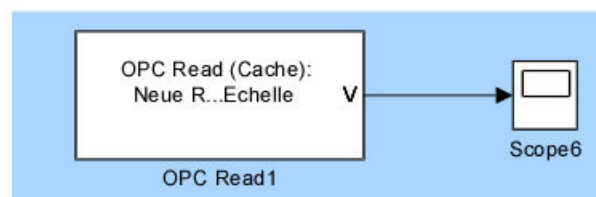

Déclaration du point de mesures (information Num) : entrée analogique

Déclaration des points de mesures (informations T.O.R) : Seuils

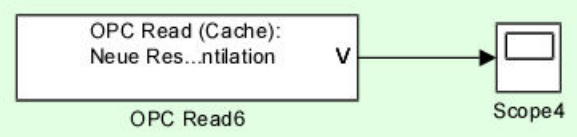

Fig. 17 : Configuration de l'acquisition sous Simulink

\footnotetext{
12 DA : Data Access

${ }^{13}$ HDA : Historical Data Access
} 
La Fig. 18 présente les résultats sous Simulink (à noter que les essais n'ont pas été menés au même moment que les essais précédents). Afin de qualifier la démarche de travail, la campagne de mesures a également été menée sur une plage temporelle de 120 secondes.

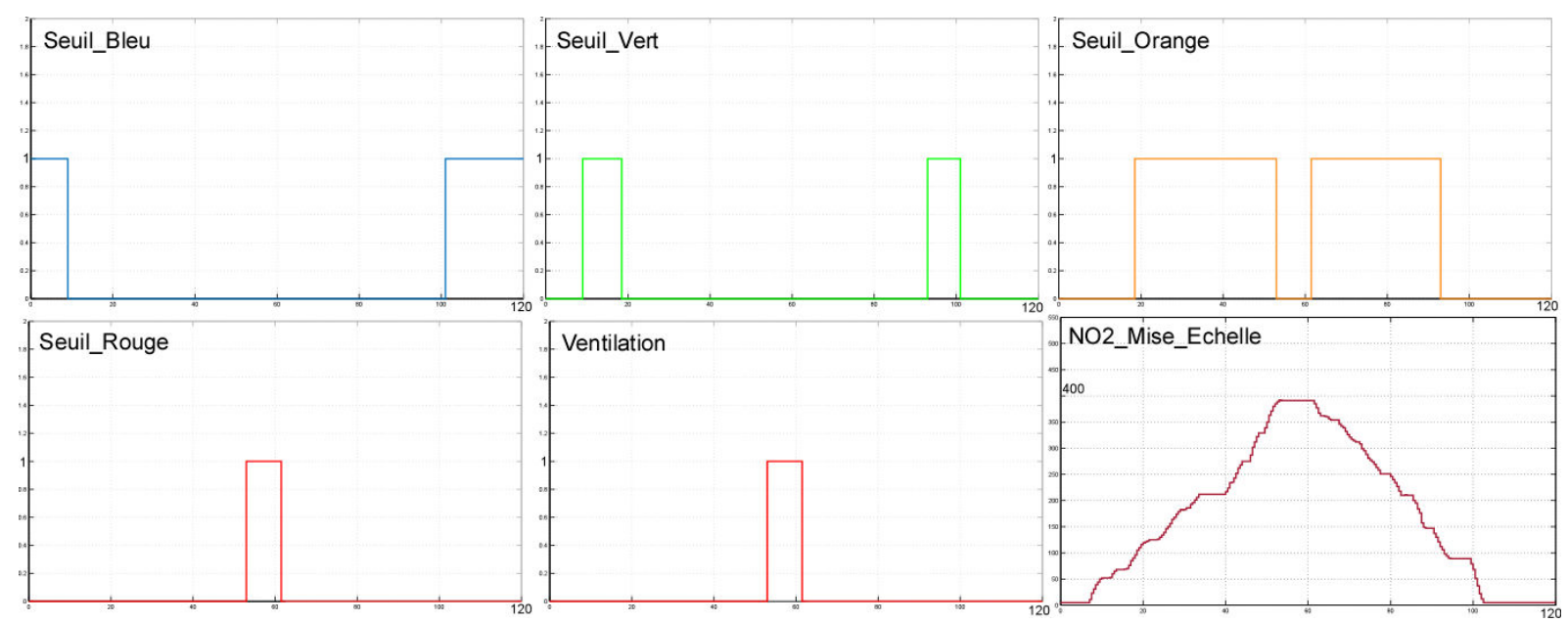

Fig. 18 : Un exemple de résultats sous Simulink

\subsection{Le client $n^{\circ} 4$}

Le client $\mathrm{n}^{\circ} 4$ fait partie des objets communicants utilisés pour des applications industrielles. Avec une administration préalable du routeur wifi (redirection de l'adresse IP de l'ordinateur connecté au réseau (Fig. 4)), la tablette pourra se connecter à différents services activés dans le client $n^{\circ} 1$. Un utilisateur peut par exemple via un navigateur Internet, visualiser l'ensemble des contenus des Items déclarés à partir du site : http://localhost/datahub.asp

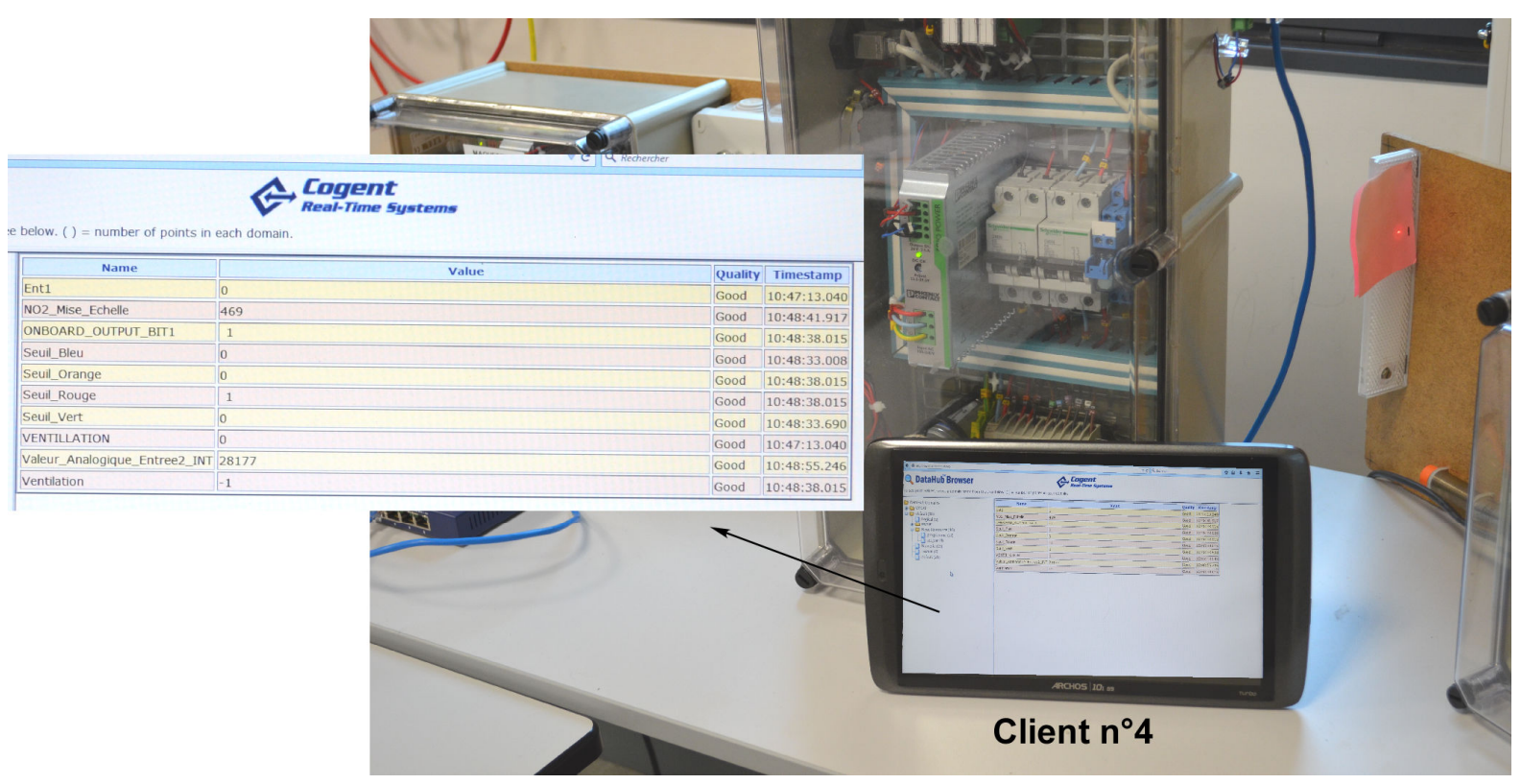

Photo 2 : Visualisation des contenus d'Items sur une tablette 


\section{CONCLUSION ET PERSPECTIVES}

Cet article tente de présenter différents aspects fondamentaux en matière d'environnement de type SCADA. La solution matérielle utilisée n'est pas classique (Bluetooth à vocation industrielle), même si les solutions opérationnelles à destination des applications industrielles sans fil sont aujourd'hui et depuis quelques années nombreuses (modem radio, pont Ethernet wireless, Zigbee, XBee Pro...).

Cette première approche de travail à vocation pédagogique dispose de plusieurs avantages : montrer et démontrer les spécificités d'un client-serveur OPC ; montrer que le logiciel Excel peut réaliser assez simplement des animations dynamiques permettant une possible orientation d'application d'Interface Homme-Machine. Ce travail permet également d'aborder la gestion des mots de passe, gestion difficile à organiser dans un monde en très grande partie numérique aujourd'hui. Néanmoins et au-delà de la configuration matérielle (sujet de travail à part entière), nous montrons que pour aboutir au résultat, il faut respecter un ensemble de procédures et de compétences liées au métier de l'informatique industrielle. Ce travail est proposé à nos étudiants de licence professionnelle Supervision des Automatismes et des Réseaux sous la forme d'un mini-projet d'une durée de 12 heures. L'intégralité de la partie matérielle est à leur disposition. L'ensemble des logiciels est installé sur 7 postes de travail (montés en réseau). Chaque binôme devra :

- développer l'application hébergée dans l'API (A), sous PC WORX (V6.0 SP2), répondant au cahier des charges de la Fig. 7 ,

- déclarer l'ensemble des Items OPC (Tableau 1),

- déclarer un client sous Cogent-DataHub,

- développer la solution de "reporting" sous Excel,

- développer un client sous Matlab,

- $\quad$ effectuer une campagne de mesures en validant l'intégralité du dispositif.

Ce sujet a été mis en place pour la première fois en 2014 où 7 binômes peuvent travailler en parallèle. C'est un sujet qui clôture une série de 3 sujets très complémentaires, consacrés aux applications actuelles en réseaux de communications industriels (solution Interbus (Phoenix Contact), solution Ethernet associée à Profibus DP accompagnée d'une traçabilité d'objets étiquetés avec des badges RFID (Siemens)). Toute la difficulté pédagogique aujourd'hui consiste à transmettre des compétences dans le domaine du savoir-faire en tentant de minimiser l'appropriation de plus en plus complexe des plateformes de développement proposées par les constructeurs.

Le travail qui vient d'être présenté est également mené avec la même approche mais sans matériel pour des étudiants de dernière année d'écoles d'ingénieurs. Pour cela, il existe un certain nombre d'émulateurs gratuits. Ces émulateurs permettent de générer des Items avec différentes caractéristiques (TOR, ASCII, NUM). Ces Items peuvent être également lus ou écris à souhait. Deux exemples validés sont presentés Fig. 19. La Fig. 19(a) concerne l'environnement KEPServeurEx de l'éditeur Kepware. La Fig. 19(b) concerne l'environnement Simulator OPC Server de l'éditeur Iconics. Des solutions génériques de l'éditeur Matrikon pourront également apporter satisfaction. 


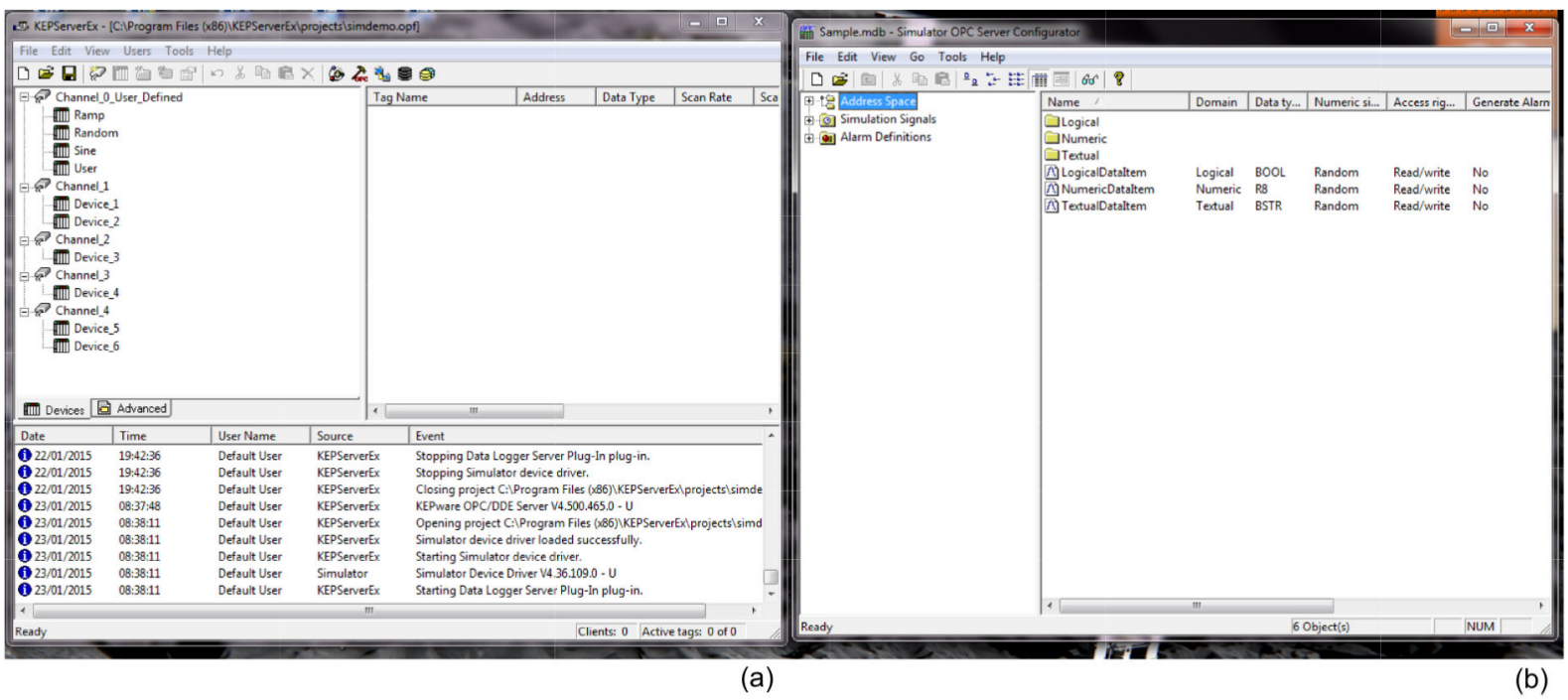

Fig. 19 : Utilisation d'émulateurs d'Items

Les perspectives sont nombreuses puisque la solution est particulièrement riche et ouverte. Le travail mené sous Excel peut aboutir à une solution relative à la création de bases de données sous Access ou My SQL... Cette ouverture vers le déploiement et l'usage d'une base de données est aujourd'hui naturelle voire obligatoire pour conforter le besoin et la nécessité de "reporting". Une solution plus conséquente d'interface Homme-machine pourra être développée et hébergée pour la tablette. Un travail plus fastidieux mais néanmoins intéressant peut être mené sur le décryptage des trames émises sur le réseau lors des échanges. Les solutions logicielles permettant de "sniffer" le réseau existent. La stabilité de Windows, du pack Office, et la robustesse des solutions sont également des éléments potentiellement bloquants et pouvant impacter le résultat final (échantillonnage des mesures, notion de besoin de temps maitrisé ou non...). Un travail pourra également être mené concernant les enjeux liés à la cyber sécurité des systèmes industriels : généralités sur les attaques, les négligences humaines, vulnérabilités des systèmes d'information, les impacts potentiels sur les systèmes...

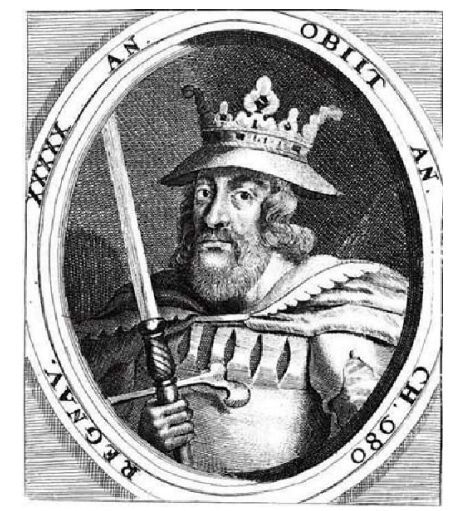

\section{Reproduction 1 : Harald Blåtand "homme à la dent bleue"}

A cent lieux de la technologie actuelle, la gourmandise pour les myrtilles de Harald $1^{\text {er }}$, surnommé Harald Blåtand "homme à la dent bleue (Reproduction 1)" nous conduit également avec ce sujet, à nous remémorer son histoire personnelle [15] : "unifier" 


\section{REFERENCES}

[1] L. Siegfried., “Industrie 4.0 - L'usine connectée - Executive summary”, Gimélec, 27 septembre 2013.

[2] M. Blanchet, R. Bergerried., "Industrie 4.0 - Les leviers de la transformation”, Gimélec, septembre, pp. 8-11, 2014.

[3] Mesures., "Communications radio : les industriels misent sur le sans fil", mesures, 805, pp. 27-40, 2008.

[4] J. Deprez, J.L. Bianchi, J. Maillefert, A. Juton.,"Bus de terrain et supervision", Société de l'électricité, de l'électronique et des technologies de l'information et de la communication, 3EI 2010-60, n60, Mars, 2010.

[5] J.P. Chemela, B. Riera., "Programmation objet pour une domotique réalisée avec un API", Actes du $10^{\text {ème }}$ Colloque sur l'Enseignement des Technologies et des Sciences de l'Information et des Systèmes (CESTIS-EEA'13), Caen (2013), pp. 78-80.

[6] P. Vrignat, M. Avila, F. Duculty, S. Begot, J.F. Millet., "Utilisation d'une architecture logicielle dans le cadre d'une mise en place d'une solution multi-clients via un serveur OPC", Gesi $\mathrm{n}^{\circ} 77$, Revue des Départements : Génie Electrique et Informatique Industrielle en IUT, 2012.

[7] P. Vrignat, F. Duculty, S. Begot, J.F Millet, M. Avila., "Développement et utilisation d'applications complémentaires dans une approche SCADA : retour sur expérience avec des étudiants BAC +2 et Bac +5 ", CETSIS, Colloque sur l'Enseignement des Technologies et des Sciences de l'Information et des Systèmes, 11ème Edition, Besançon, France, pp. 76-81, 2014.

[8] B. Riera, F. Gellot, O. Dubois, J.P. Chemla et S. Triki., "L'utilisation pédagogique et l'enseignement des TIC dans les automatismes", J3eA, Journal sur l'enseignement des sciences et technologies de l'information et des systèmes, Volume 4, Hors-Série 2, 2005.

[9] Communication Radio., "Le sans-fil étend son influence", Mesures 843, pp. 22-26, Mars, 2012.

[10] http://www.iconics.com/Home.aspx

[11] http://www.cogentdatahub.com/

[12] ObsAirvatoire., "Bilan 2011 de la qualité de l'air à proximité du Duplex A86”, AIRP ARIFSurveillance de la Qualité de l'Air en Ile-de-France", mars, 2012.

[13] P. Vrignat., "Contrôle Commande et Supervision Son évolution : ses enjeux, les nouvelles tendances et nouveaux services”, Rapport de Master, Université d'Orléans, 2007.

[14] Guidance for Industry, 21 CFR Part 11: Electronic Records; Electronic Signatures Validation, U.S. Department of Health and Human Services, Food and Drug Administration, 2001.

[15] http://fr.wikipedia.org/wiki/Harald_Ier_de_Danemark 